\title{
Relationships between Mahler Expansion and Higher Order q-Daehee polynomials
}

\author{
Ugur Duran ${ }^{1, *}$ and Mehmet Acikgoz ${ }^{2}$ \\ ${ }^{1}$ Department of the Basic Concepts of Engineering, \\ Faculty of Engineering and Natural Sciences, \\ Iskenderun Technical University, TR-31200 Hatay, Turkey \\ E-Mail: mtdrnugur@gmail.com8jugur.duran@iste.edu.tr \\ ${ }^{*}$ Corresponding Author \\ ${ }^{2}$ University of Gaziantep, Faculty of Science and Arts, \\ Department of Mathematics, TR-27310 Gaziantep, Turkey \\ E-Mail: acikgoz@gantep.edu.tr
}

\begin{abstract}
In this paper, we derive multifarious relationships among the two types of higher order $q$-Daehee polynomials and $p$-adic gamma function via Mahler theorem. Also, we compute some weighted $p$-adic $q$-integrals of the derivative of $p$-adic gamma function related to the Stirling numbers of the both kinds and the $q$-Bernoulli polynomials of order $k$.
\end{abstract}

2010 Mathematics Subject Classification. Primary 05A10, 05A30; Secondary 11B65, 11S80, 33B15. Key Words and Phrases. $q$-numbers, $p$-adic numbers, $p$-adic gamma function, Mahler expansion, Bernoulli polynomials, Daehee polynomials, Stirling numbers of the first kind, Stirling numbers of the second kind.

\section{INTRODUCTION}

The $p$-adic numbers are a counterintuitive arithmetic system, which were primarily considered by the Kummer in circa 1850. With the consideration of these numbers, many mathematicians and physicists started to develop new scientific tools using their available, useful and applicable properties. Firstly, Kurt Hensel who is a German mathematician (1861-1941) advanced the $p$-adic numbers in a study associated with the improvement of algebraic numbers in power series in about 1897. Several effects of these researches have emerged in mathematics and physics such as $p$-adic analysis, string theory, $p$-adic quantum mechanics, quantum field theory, representation theory, algebraic geometry, complex systems, dynamical systems, genetic codes and so on ( $c f$. [1-4,6-17]; also see the references cited in each of these earlier studies). The one important tool of the mentioned advancements is $p$-adic gamma function which is introduced by Yasou Morita [14] in 1975. Intense research activities in such an area as $p$-adic gamma function is principally motivated by their significance in $p$-adic analysis. Hereby, in recent fourty years, $p$-adic gamma function and its diverse extensions have been studied and progressed broadly by many mathematicians, $c f .[4,6-8,13,14,16]$; see also the related references cited therein.

The Daehee polynomials, denoted by $D_{n}(x)$, are defined by means of the following exponential generating function (cf. [10]):

$$
\sum_{n=0}^{\infty} D_{n}(x) \frac{t^{n}}{n !}=\frac{\log (1+t)}{t}(1+t)^{x} .
$$

In the case $x=0$ in $(1.1)$, one can get $D_{n}(0):=D_{n}$ standing for $n$-th Daehee number, see $[2,4,9,10,15,17]$ for more detailed information about these related issues.

Throughtout this paper, we shall use the following figures: $\mathbb{Z}$ indicates the set of all integers, $\mathbb{Q}$ indicates the field of rational numbers, $\mathbb{Z}_{p}$ indicates the ring of the $p$-adic integers, $\mathbb{Q}_{p}$ indicates the field of the $p$-adic 
numbers, and $\mathbb{C}_{p}$ indicates the $p$-adic completion of an algebraic closure of $\mathbb{Q}_{p}$, where $p$ be a fixed prime number $(c f .[1-4,6-17])$. Let $\mathbb{N}=\{1,2,3, \cdots\}$ and $\mathbb{N}^{*}=\mathbb{N} \cup\{0\}$.

The familiar $p$-adic Haar distribution $\mu_{0}$ and the Volkenborn integral ( $p$-adic integral) $I_{0}(f)$ of a function

$$
f \in U D\left(\mathbb{Z}_{p}, \mathbb{C}_{p}\right)=\left\{f \mid f: \mathbb{Z}_{p} \rightarrow \mathbb{C}_{p} \text { is uniformly differentiable function }\right\},
$$

respectively, are given by (cf. $[2,4,6,7,9-12,15-17])$

$$
\mu_{0}\left(y+p^{N} \mathbb{Z}_{p}\right)=1 / p^{N}
$$

and

$$
I_{0}(f)=\int_{\mathbb{Z}_{p}} f(x) d \mu_{0}(x)=\lim _{N \rightarrow \infty} \frac{1}{p^{N}} \sum_{x=0}^{p^{N}-1} f(x)
$$

which yields the Daehee polynomials $D_{n}(x)$ and Daehee numbers $D_{n}$, for $n \in \mathbb{N}^{*}$, as follows

$$
D_{n}(x)=\int_{\mathbb{Z}_{p}}(x+y)_{n} d \mu_{0}(y) \text { and } D_{n}=\int_{\mathbb{Z}_{p}}(y)_{n} d \mu_{0}(y),
$$

where the symbol $(x)_{n}$ denotes the falling factorial given by (cf. $\left.[2,4,9,10,15,17]\right)$

$$
(x)_{n}=x(x-1)(x-2) \cdots(x-n+1) .
$$

The falling factorial $(x)_{n}$ satisfies the following identity:

$$
(x)_{n}=\sum_{k=0}^{n} S_{1}(n, k) x^{k},
$$

where $S_{1}(n, k)$ denotes the Stirling number of the first kind (see $[2,4,9,10,15,17]$ ).

The notation $q$ may be varyingly considered as indeterminate, complex number $q \in \mathbb{C}$ with $0<|q|<1$, or $p$-adic number $q \in \mathbb{C}_{p}$ with $|q-1|_{p}<p^{-\frac{1}{p-1}}$ so that $q^{x}=\exp (x \log q)$ for $|x|_{p} \leq 1$, where $|\cdot|_{p}$ indicates the $p$-adic norm on $\mathbb{C}_{p}$ normalized by $|p|_{p}=1 / p$.

The $q$-Volkenborn integral or $p$-adic $q$-integral on $\mathbb{Z}_{p}$ of a function $f \in U D\left(\mathbb{Z}_{p}\right)$ is defined $(c f .[2,4,6,9,13,15])$ as follows:

$$
I_{q}(f)=\int_{\mathbb{Z}_{p}} f(x) d \mu_{q}(x)=\lim _{N \rightarrow \infty} \frac{1}{\left[p^{N}\right]_{q}} \sum_{x=0}^{p^{N}-1} f(x) q^{x} .
$$

Suppose that $f_{1}(x)=f(x+1)$. Then, we see that

$$
q I_{q}\left(f_{1}\right)=I_{q}(f)+(q-1) f(0)+\frac{q-1}{\log q} f^{\prime}(0) .
$$

For these related issues, see $[2,4,6,9,13,15]$ and related references cited therein.

The higher order $q$-Daehee numbers $D_{n, q}^{(k)}$ and higher order $q$-Daehee polynomials $D_{n, q}^{(k)}(x)$ are defined $(c f$. [2]) by means of $q$-Volkenborn integrals for $k \in \mathbb{N}$ :

$$
\begin{aligned}
D_{n, q}^{(k)} & =\int_{\mathbb{Z}_{p}} \cdots \int_{\mathbb{Z}_{p}}\left(x_{1}+\cdots+x_{k}\right)_{n} d \mu_{q}\left(x_{1}\right) \cdots d \mu_{q}\left(x_{k}\right) \quad(n \geq 0), \\
D_{n, q}^{(k)}(x) & =\int_{\mathbb{Z}_{p}} \cdots \int_{\mathbb{Z}_{p}}\left(x_{1}+\cdots+x_{k}+x\right)_{n} d \mu_{q}\left(x_{1}\right) \cdots d \mu_{q}\left(x_{k}\right) \quad(n \geq 0) .
\end{aligned}
$$

When $k=1$, it is obvious that $\lim _{q \rightarrow 1} D_{n, q}^{(1)}:=D_{n}$ and $\lim _{q \rightarrow 1} D_{n, q}^{(1)}(x):=D_{n}(x)$.

The higher order $q$-Daehee numbers and polynomials of the second kind are introduced by the following $q$-Volkenborn integrals:

$$
\begin{aligned}
\widehat{D}_{n, q}^{(k)} & =\int_{\mathbb{Z}_{p}} \cdots \int_{\mathbb{Z}_{p}}\left(-x_{1}-\cdots-x_{k}\right)_{n} d \mu_{q}\left(x_{1}\right) \cdots d \mu_{q}\left(x_{k}\right) \quad(n \geq 0), \\
\widehat{D}_{n, q}^{(k)}(x) & =\int_{\mathbb{Z}_{p}} \cdots \int_{\mathbb{Z}_{p}}\left(-x_{1}-\cdots-x_{k}+x\right)_{n} d \mu_{q}\left(x_{1}\right) \cdots d \mu_{q}\left(x_{k}\right) \quad(n \geq 0) .
\end{aligned}
$$


The $q$-Daehee polynomials and numbers and their various generalizations have been studied by many mathematicians, $c f .[2,4,6,9,13,15]$; see also the related references cited therein.

In the present paper, we are interested in constructing the correlations between the $p$-adic gamma function and the higher order $q$-Daehee polynomials and numbers by using the methods of the $q$-Volkenborn integral and Mahler series expansion; in the last part, we examine the results derived in this paper.

\section{Higher Order q-Daehee Polynomials Associated with Mahler Theorem}

This section provides some properties, identities and correlations for the mentioned gamma function, the higher order $q$-Daehee polynomials and numbers of the both types, Stirling numbers of the first kind and $p$-adic Euler constant by using the Mahler expansion of the $p$-adic gamma function given by Proposition 1 below.

The $p$-adic gamma function is defined as follows

$$
\Gamma_{p}(x)=\lim _{n \rightarrow x}(-1)^{n} \prod_{\substack{j<n \\(p, j)=1}} j \quad\left(x \in \mathbb{Z}_{p}\right),
$$

where $n$ approaches $x$ through positive integers.

The $p$-adic gamma function in conjunction with its several extensions and $p$-adic Euler constant have been developed by many physicists and mathematicians, $c f .[4,6-8,13,14,16]$; see also the references cited in each of these earlier works.

For $x \in \mathbb{Z}_{p}$, the symbol $\left(\begin{array}{l}x \\ n\end{array}\right)$ is given by $\left(\begin{array}{l}x \\ n\end{array}\right)=\frac{x(x-1) \cdots(x-n+1)}{n !}(n \in \mathbb{N})$ with $\left(\begin{array}{l}x \\ 0\end{array}\right)=1$.

Let $x \in \mathbb{Z}_{p}$ and $n \in \mathbb{N}$. The functions $x \rightarrow\left(\begin{array}{l}x \\ n\end{array}\right)$ form an orthonormal base of the space $C\left(\mathbb{Z}_{p} \rightarrow \mathbb{C}_{p}\right)$ with respect to the Euclidean norm $\|\cdot\|_{\infty}$. The mentioned orthonormal base satisfies the following equality:

$$
\left(\begin{array}{l}
x \\
n
\end{array}\right)^{\prime}=\sum_{j=0}^{n-1} \frac{(-1)^{n-j-1}}{n-j}\left(\begin{array}{l}
x \\
j
\end{array}\right) \quad(\text { see }[6,7,13,16]) .
$$

Mahler investigated a generalization for continuous maps of a $p$-adic variable utilizing the special polynomials as binomial coefficient polynomial [12] in 1958. It implies that for any $f \in C\left(\mathbb{Z}_{p} \rightarrow \mathbb{C}_{p}\right)$, there exist unique elements $a_{0}, a_{1}, a_{2}, \ldots$ of $\mathbb{C}_{p}$ such that

$$
f(x)=\sum_{n=0}^{\infty} a_{n}\left(\begin{array}{l}
x \\
n
\end{array}\right) \quad\left(x \in \mathbb{Z}_{p}\right) .
$$

The base $\left\{\left(\begin{array}{l}* \\ n\end{array}\right): n \in \mathbb{N}\right\}$ is named as Mahler base of the space $C\left(\mathbb{Z}_{p} \rightarrow \mathbb{C}_{p}\right)$, and the components $\left\{a_{n}: n \in \mathbb{N}\right\}$ in $f(x)=\sum_{n=0}^{\infty} a_{n}\left(\begin{array}{l}x \\ n\end{array}\right)$ are called Mahler coefficients of $f \in C\left(\mathbb{Z}_{p} \rightarrow \mathbb{C}_{p}\right)$. The Mahler expansion of the $p$-adic gamma function $\Gamma_{p}$ and its Mahler coefficients are determined in [16] as follows.

Proposition 1. For $x \in \mathbb{Z}_{p}$, let $\Gamma_{p}(x+1)=\sum_{n=0}^{\infty} a_{n}\left(\begin{array}{l}x \\ n\end{array}\right)$ be Mahler series of $\Gamma_{p}$. Then its coefficients satisfy the following identity:

$$
\sum_{n \geqq 0}(-1)^{n+1} a_{n} \frac{x^{n}}{n !}=\frac{1-x^{p}}{1-x} \exp \left(x+\frac{x^{p}}{p}\right) .
$$

We now provide the higher order $q$-Volkenborn integral of the $p$-adic gamma function via the higher order $q$-Daehee polynomials by Theorem 1 .

Theorem 1. Let $x, x_{i} \in \mathbb{Z}_{p}$ where $i \in\{1,2, \ldots k\}$. We have

$$
\int_{\mathbb{Z}_{p}} \cdots \int_{\mathbb{Z}_{p}} \Gamma_{p}\left(x_{1}+\cdots+x_{k}+x+1\right) d \mu_{q}\left(x_{1}\right) \cdots d \mu_{q}\left(x_{k}\right)=\sum_{n=0}^{\infty} a_{n} \frac{D_{n, q}^{(k)}(x)}{n !},
$$

where $a_{n}$ is given by Proposition 1. 
Proof. For $x, x_{i} \in \mathbb{Z}_{p}$ where $i \in\{1,2, \ldots k\}$, by using the relation $\left(\begin{array}{c}x_{1}+x_{2} \\ n\end{array}\right)=\frac{\left(x_{1}+x_{2}\right)_{n}}{n !}$ and Proposition 1 , we get

$$
\begin{aligned}
& \int_{\mathbb{Z}_{p}} \cdots \int_{\mathbb{Z}_{p}} \Gamma_{p}\left(x_{1}+\cdots+x_{k}+x+1\right) d \mu_{q}\left(x_{1}\right) \cdots d \mu_{q}\left(x_{k}\right) \\
= & \int_{\mathbb{Z}_{p}} \cdots \int_{\mathbb{Z}_{p}} \sum_{n=0}^{\infty} a_{n} \frac{\left(x_{1}+\cdots+x_{k}+x\right)_{n}}{n !} d \mu_{q}\left(x_{1}\right) \cdots d \mu_{q}\left(x_{k}\right) \\
= & \sum_{n=0}^{\infty} a_{n} \frac{1}{n !} \int_{\mathbb{Z}_{p}} \cdots \int_{\mathbb{Z}_{p}}\left(x_{1}+\cdots+x_{k}+x\right)_{n} d \mu_{q}\left(x_{1}\right) \cdots d \mu_{q}\left(x_{k}\right),
\end{aligned}
$$

which is the desired result (2.5) via the formula (1.9).

We now examine a consequence of the Theorem 1 as follows.

Corollary 1. Choosing $x=0$ in Theorem 1 gives the following relation for $\Gamma_{p}$ and $D_{n, q}^{(k)}$ :

$$
\int_{\mathbb{Z}_{p}} \cdots \int_{\mathbb{Z}_{p}} \Gamma_{p}\left(x_{1}+\cdots+x_{k}+1\right) d \mu_{q}\left(x_{1}\right) \cdots d \mu_{q}\left(x_{k}\right)=\sum_{n=0}^{\infty} a_{n} \frac{D_{n, q}^{(k)}}{n !},
$$

where $a_{n}$ is given by Proposition 1.

Let $n \in \mathbb{N}_{0}$ and $k \in \mathbb{N}$. Cho et al. [2] gave the following correlation:

$$
D_{n, q}^{(k)}(x)=\sum_{m=0}^{n} S_{1}(n, m) B_{m, q}^{(k)}(x),
$$

where $B_{m, q}^{(k)}(x)$ denotes the $m$-th Bernoulli polynomials of order $k$ defined by

$$
B_{m, q}^{(k)}(x)=\int_{\mathbb{Z}_{p}} \cdots \int_{\mathbb{Z}_{p}}\left(x_{1}+\cdots+x_{k}+x\right)^{m} d \mu_{q}\left(x_{1}\right) \cdots d \mu_{q}\left(x_{k}\right) \quad\left(m \in \mathbb{N}_{0}\right) .
$$

As a result of Theorem 1 and relation (2.7), one other higher order $q$-Volkenborn integrals of the $p$-adic gamma function via the $q$-Bernoulli polynomials of order $k$ is stated below.

Corollary 2. For $x, x_{i} \in \mathbb{Z}_{p}$ where $i \in\{1,2, \ldots k\}$, we acquire

$$
\int_{\mathbb{Z}_{p}} \cdots \int_{\mathbb{Z}_{p}} \Gamma_{p}\left(x_{1}+\cdots+x_{k}+x+1\right) d \mu_{q}\left(x_{1}\right) \cdots d \mu_{q}\left(x_{k}\right)=\sum_{n=0}^{\infty} \sum_{m=0}^{n} a_{n} \frac{S_{1}(n, m)}{n !} B_{m, q}^{(k)}(x) .
$$

Here is the $p$-adic $q$-integrals of the derivative of the $p$-adic gamma function.

Theorem 2. For $x, x_{i} \in \mathbb{Z}_{p}$ where $i \in\{1,2, \ldots k\}$, we have

$$
\int_{\mathbb{Z}_{p}} \cdots \int_{\mathbb{Z}_{p}} \Gamma_{p}^{\prime}\left(x_{1}+\cdots+x_{k}+x+1\right) d \mu_{q}\left(x_{1}\right) \cdots d \mu_{q}\left(x_{k}\right)=\sum_{n=0}^{\infty} \sum_{j=0}^{n-1} a_{n} \frac{(-1)^{n-j-1} D_{j, q}^{(k)}(x)}{(n-j) j !} .
$$

Proof. In view of Proposition 1, we obtain

$$
\begin{aligned}
& \int_{\mathbb{Z}_{p}} \cdots \int_{\mathbb{Z}_{p}} \Gamma_{p}^{\prime}\left(x_{1}+\cdots+x_{k}+x+1\right) d \mu_{q}\left(x_{1}\right) \cdots d \mu_{q}\left(x_{k}\right) \\
= & \int_{\mathbb{Z}_{p}} \cdots \int_{\mathbb{Z}_{p}} \sum_{n=0}^{\infty} a_{n}\left(\begin{array}{c}
x_{1}+\cdots+x_{k}+x \\
n
\end{array}\right)^{\prime} d \mu_{q}\left(x_{1}\right) \cdots d \mu_{q}\left(x_{k}\right) \\
= & \sum_{n=0}^{\infty} a_{n} \int_{\mathbb{Z}_{p}} \cdots \int_{\mathbb{Z}_{p}}\left(\begin{array}{c}
x_{1}+\cdots+x_{k}+x \\
n
\end{array}\right)^{\prime} d \mu_{q}\left(x_{1}\right) \cdots d \mu_{q}\left(x_{k}\right)
\end{aligned}
$$


and using (2.2), we derive

$$
\begin{aligned}
& \int_{\mathbb{Z}_{p}} \cdots \int_{\mathbb{Z}_{p}} \Gamma_{p}^{\prime}\left(x_{1}+\cdots+x_{k}+x+1\right) d \mu_{q}\left(x_{1}\right) \cdots d \mu_{q}\left(x_{k}\right) \\
= & \sum_{n=0}^{\infty} \sum_{j=0}^{n-1} a_{n} \frac{(-1)^{n-j-1}}{n-j} \int_{\mathbb{Z}_{p}} \cdots \int_{\mathbb{Z}_{p}}\left(\begin{array}{c}
x_{1}+\cdots+x_{k}+x \\
j
\end{array}\right) d \mu_{q}\left(x_{1}\right) \cdots d \mu_{q}\left(x_{k}\right) \\
= & \sum_{n=0}^{\infty} \sum_{j=0}^{n-1} a_{n} \frac{(-1)^{n-j-1}}{n-j} \frac{D_{j, q}^{(k)}(x)}{j !} .
\end{aligned}
$$

The immediate result of Theorem 2 is given as follows.

Corollary 3. For $x_{i} \in \mathbb{Z}_{p}$ where $i \in\{1,2, \ldots k\}$, we have

$$
\int_{\mathbb{Z}_{p}} \cdots \int_{\mathbb{Z}_{p}} \Gamma_{p}^{\prime}\left(x_{1}+\cdots+x_{k}+1\right) d \mu_{q}\left(x_{1}\right) \cdots d \mu_{q}\left(x_{k}\right)=\sum_{n=0}^{\infty} \sum_{j=0}^{n-1} a_{n} \frac{(-1)^{n-j-1} D_{j, q}^{(k)}}{(n-j) j !} .
$$

We state the following theorem including a relation between $\Gamma_{p}(x)$ and $\widehat{D}_{n, q}^{(k)}(x)$.

Theorem 3. For $x, x_{i} \in \mathbb{Z}_{p}$ where $i \in\{1,2, \ldots k\}$, we have

$$
\int_{\mathbb{Z}_{p}} \cdots \int_{\mathbb{Z}_{p}} \Gamma_{p}\left(-x_{1}-\cdots-x_{k}-x+1\right) d \mu_{q}\left(x_{1}\right) \cdots d \mu_{q}\left(x_{k}\right)=\sum_{n=0}^{\infty} a_{n} \frac{\widehat{D}_{n, q}^{(k)}(x)}{n !},
$$

where $a_{n}$ is given by Proposition 1.

Proof. For $x, x_{i} \in \mathbb{Z}_{p}$ where $i$ lies in $\{1,2, \ldots k\}$, by utilizing the relation $\left(\begin{array}{c}-x_{1}-x_{2} \\ n\end{array}\right)=\frac{\left(-x_{1}-x_{2}\right)_{n}}{n !}$ and Proposition 1 , we get

$$
\begin{aligned}
& \int_{\mathbb{Z}_{p}} \cdots \int_{\mathbb{Z}_{p}} \Gamma_{p}\left(-x_{1}-\cdots-x_{k}+x+1\right) d \mu_{q}\left(x_{1}\right) \cdots d \mu_{q}\left(x_{k}\right) \\
= & \int_{\mathbb{Z}_{p}} \cdots \int_{\mathbb{Z}_{p}} \sum_{n=0}^{\infty} a_{n} \frac{\left(-x_{1}-\cdots-x_{k}+x\right)_{n}}{n !} d \mu_{q}\left(x_{1}\right) \cdots d \mu_{q}\left(x_{k}\right) \\
= & \sum_{n=0}^{\infty} a_{n} \frac{1}{n !} \int_{\mathbb{Z}_{p}} \cdots \int_{\mathbb{Z}_{p}}\left(-x_{1}-\cdots-x_{k}+x\right)_{n} d \mu_{q}\left(x_{1}\right) \cdots d \mu_{q}\left(x_{k}\right),
\end{aligned}
$$

which is the desired result with (1.11).

A consequence of Theorem 3 is given by the following corollary.

Corollary 4. Upon setting $x=0$ in Theorem 3 gives the following relation for $\Gamma_{p}$ and $\widehat{D}_{n, q}^{(k)}$ :

$$
\int_{\mathbb{Z}_{p}} \cdots \int_{\mathbb{Z}_{p}} \Gamma_{p}\left(-x_{1}-\cdots-x_{k}+1\right) d \mu_{q}\left(x_{1}\right) \cdots d \mu_{q}\left(x_{k}\right)=\sum_{n=0}^{\infty} a_{n} \frac{\widehat{D}_{n, q}^{(k)}}{n !},
$$

where $a_{n}$ is given by Proposition 1.

Let $n \in \mathbb{N}_{0}$ and $k \in \mathbb{N}$. Cho et al. [2] gave the following correlation:

$$
\widehat{D}_{n, q}^{(k)}(x)=\sum_{m=0}^{n}(-1)^{n-m} S_{1}(n, m) B_{m, q}^{(k)}(-x),
$$

which yields the following result with the help of Theorem 3 . 
Corollary 5. For $x, x_{i} \in \mathbb{Z}_{p}$ where $i \in\{1,2, \ldots k\}$, we acquire

$$
\int_{\mathbb{Z}_{p}} \cdots \int_{\mathbb{Z}_{p}} \Gamma_{p}\left(-x_{1}-\cdots-x_{k}+x+1\right) d \mu_{q}\left(x_{1}\right) \cdots d \mu_{q}\left(x_{k}\right)=\sum_{n=0}^{\infty} \sum_{m=0}^{n}(-1)^{n-m} a_{n} \frac{S_{1}(n, m)}{n !} B_{m, q}^{(k)}(-x) .
$$

We observe that

$$
\begin{aligned}
(-y)_{n} & =(-y)(-y+1) \cdots(-y-n+1) \\
& =(-1)^{n} y(y-1) \cdots(y+n-1) \\
& =(-1)^{n} \sum_{m=0}^{n} S_{2}(n, m) y^{m},
\end{aligned}
$$

where $S_{2}(n, m)$ denotes the Stirling numbers of the second kind, $c f$. $[2,4,9,10,15,17]$, defined by the following exponential generating function

$$
\frac{\left(e^{t}-1\right)^{m}}{m !}=\sum_{n \geq 0} S_{2}(n, m) \frac{t^{n}}{n !}
$$

We lastly state one other $p$-adic $q$-integrals of the derivative of the $p$-adic gamma function by means of the $q$-Bernoulli polynomials of order $k$ and the Stirling numbers of the second kind.

Theorem 4. For $x, x_{i} \in \mathbb{Z}_{p}$ where $i \in\{1,2, \ldots k\}$, we have

$$
\int_{\mathbb{Z}_{p}} \cdots \int_{\mathbb{Z}_{p}} \Gamma_{p}^{\prime}\left(-x_{1}-\cdots-x_{k}-x+1\right) d \mu_{q}\left(x_{1}\right) \cdots d \mu_{q}\left(x_{k}\right)=\sum_{n=0}^{\infty} \sum_{j=0}^{n-1} \sum_{m=0}^{j} S_{2}(j, m) a_{n} \frac{(-1)^{n-1}}{n-j} \frac{B_{j, q}^{(k)}(-x)}{j !} .
$$

Proof. Via Proposition 1, we get

$$
\begin{aligned}
& \int_{\mathbb{Z}_{p}} \cdots \int_{\mathbb{Z}_{p}} \Gamma_{p}^{\prime}\left(-x_{1}-\cdots-x_{k}+x+1\right) d \mu_{q}\left(x_{1}\right) \cdots d \mu_{q}\left(x_{k}\right) \\
= & \int_{\mathbb{Z}_{p}} \cdots \int_{\mathbb{Z}_{p}} \sum_{n=0}^{\infty} a_{n}\left(\begin{array}{c}
-x_{1}-\cdots-x_{k}+x \\
n
\end{array}\right)^{\prime} d \mu_{q}\left(x_{1}\right) \cdots d \mu_{q}\left(x_{k}\right) \\
= & \sum_{n=0}^{\infty} a_{n} \int_{\mathbb{Z}_{p}} \cdots \int_{\mathbb{Z}_{p}}\left(\begin{array}{c}
-x_{1}-\cdots-x_{k}+x \\
n
\end{array}\right)^{\prime} d \mu_{q}\left(x_{1}\right) \cdots d \mu_{q}\left(x_{k}\right)
\end{aligned}
$$

and utilizing (2.2) and (2.8), we derive

$$
\begin{aligned}
& \int_{\mathbb{Z}_{p}} \cdots \int_{\mathbb{Z}_{p}} \Gamma_{p}^{\prime}\left(-x_{1}-\cdots-x_{k}+x+1\right) d \mu_{q}\left(x_{1}\right) \cdots d \mu_{q}\left(x_{k}\right) \\
= & \sum_{n=0}^{\infty} \sum_{j=0}^{n-1} a_{n} \frac{(-1)^{n-j-1}}{n-j} \int_{\mathbb{Z}_{p}} \cdots \int_{\mathbb{Z}_{p}}\left(\begin{array}{c}
-x_{1}-\cdots-x_{k}+x \\
j
\end{array}\right) d \mu_{q}\left(x_{1}\right) \cdots d \mu_{q}\left(x_{k}\right) \\
= & \sum_{n=0}^{\infty} \sum_{j=0}^{n-1} a_{n} \frac{(-1)^{n-j-1}}{(n-j) j !} \int_{\mathbb{Z}_{p}} \cdots \int_{\mathbb{Z}_{p}}\left(-x_{1}-\cdots-x_{k}+x\right)_{j} d \mu_{q}\left(x_{1}\right) \cdots d \mu_{q}\left(x_{k}\right) \\
= & \sum_{n=0}^{\infty} \sum_{j=0}^{n-1} a_{n} \frac{(-1)^{n-j-1}}{(n-j) j !} \int_{\mathbb{Z}_{p}} \cdots \int_{\mathbb{Z}_{p}}(-1)^{j} \sum_{m=0}^{j} S_{2}(j, m)\left(x_{1}+\cdots+x_{k}-x\right)^{m} d \mu_{q}\left(x_{1}\right) \cdots d \mu_{q}\left(x_{k}\right) \\
= & \sum_{n=0}^{\infty} \sum_{j=0}^{n-1} \sum_{m=0}^{j} S_{2}(j, m) a_{n} \frac{(-1)^{n-1}}{n-j} \frac{B_{j, q}^{(k)}(-x)}{j !} .
\end{aligned}
$$




\section{Conclusion and Observations}

In the present paper, by means of the higher order $q$-Daehee numbers and polynomials, we have discovered some $q$-Volkenborn integrals of $p$-adic gamma function via their Mahler expansions. The calculations of the $p$-adic gamma function have been derived in terms of the higher order $q$-Daehee polynomials and numbers and higher order $q$-Daehee polynomials and numbers of the second kind. Moreover, we have investigated higher order $q$-Volkenborn integral of the derivative of $p$-adic gamma function. The approach given in this paper is general and can be made by using the different $p$-adic integral and choosing the appropriate polynomials and numbers. Some results derived in this paper reduce to the results in the paper [4].

Competing Interests. The authors declare that they have no competing interests.

Authors' Contributions. The authors declare that the work was realized in collaboration with the same responsibility. All authors read and approved the final manuscript.

\section{References}

[1] S. Araci, E. Ağyüz, M. Acikgoz, On a $q$-analog of some numbers and polynomials, J. Inequal. Appl., 19,2015 doi: 10.1186/s13660-014-0542-y.

[2] Y.K. Cho, T. Kim, T. Mansour, S.-H. Rim, Higher order $q$-Daehee polynomials, J. Comput. Anal. Appl., 19 (1), 2015 , 167-173.

[3] J. Diamond, The $p$-adic log gamma function and $p$-adic Euler constant, Trans. Amer. Math. Soc., 233, 1977, 321-337.

[4] U. Duran, M. Acikgoz, On $p$-adic gamma function related to $q$-Daehee polynomials and numbers, accepted for publication in Proyecciones J. Math., 2018.

[5] B. N. Guo, F. Qi, Some identities and an explicit formula for Bernoulli and Stirling numbers, Anal. Appl., 19 (1), 2015 , 167-173.

[6] Ö. Ç. Havara and H. Menken, On the Volkenborn integral of the $q$-extension of the $p$-adic gamma function, J. Math. Anal., 8 (2), 2017, 64-72.

[7] Ö. Ç. Havare and H. Menken, The Volkenborn integral of the p-adic gamma function, Int. J. Adv. Appl. Funct., 5 (2), 2018, 56-59.

[8] Y. S. Kim, $q$-analogues of $p$-adic gamma functions and $p$-adic Euler constants, Comm. Korean Math. Soc., 13 (4), 1998 , 735-741.

[9] T. Kim, S.-H. Lee, T. Mansour and J.-J. Seo, A note on $q$-Daehee polynomials and numbers, Adv. Stud. Contemp. Math., 24 (2), 2014, 155-160.

[10] D. S. Kim and T. Kim, Daehee numbers and polynomials, Appl. Math. Sci., 7 (120), 2013, 5969-5976.

[11] N. Koblitz, $p$-adic numbers, $p$-adic analysis, and Zeta functions (Springer-Verlag, New York Inc, 1977).

[12] K. Mahler, An interpolation series for continuous functions of a $p$-adic variable, J. Reine Angew. Math., $199,1958,23-34$.

[13] H. Menken, A. Körükçü, Some properties of the $q$-extension of the $p$-adic gamma function, Abst. Appl. Anal., Volume 2013, Article ID 176470, 4 pages.

[14] Y. Morita, A p-adic analogue of the $\Gamma$-function, J. Fac. Sci., 22 (2), 1975, 255-266.

[15] H. Ozden, I. N. Cangul, Y. Simsek, Remarks on $q$-Bernoulli numbers associated with Daehee numbers, Adv. Stud. Contemp. Math., 18 (1), 2009, 41-48.

[16] A. M. Robert, A course in $p$-adic analysis (Springer-Verlag New York, Inc., 2000).

[17] Y. Simsek, Apostol type Daehee numbers and polynomials, Adv. Stud. Contemp. Math., 26 (3), 2016, 555-566. 